\title{
Thymic dendritic cells traffic to thymi of allogeneic recipients and prolong graft survival
}

\author{
Steven R. Duncan, Nickolas G. Capetanakis, Brian R. Lawson, \\ and Argyrios N. Theofilopoulos
}

Department of Immunology, The Scripps Research Institute, La Jolla, California, USA

Address correspondence to: Steven R. Duncan, Pulmonary, Allergy, and Critical Care Medicine, University of Pittsburgh, NW 628 Montefiore University Hospital, 3459 Fifth Avenue, Pittsburgh, Pennsylvania 15213, USA.

Phone: (412) 692-2210; Fax: (412) 692-2260; E-mail: duncsr@msx.upmc.edu.

Received for publication January 5, 2001, and accepted in revised form January 28, 2002.

We have demonstrated that murine thymic dendritic cells (DCs) isolated from donor mice have the capability to home to thymi of fully allogeneic recipients after intravenous injections, where they induce $T$ cell deletions and prolong donor-strain airway and skin graft survival. In contrast, infused splenic DCs immigrated poorly to thymi, and did not affect graft survival. These findings suggest that preferential homing may be an important mechanistic difference among subpopulations of DCs that mediate immune functions and illustrate a novel methodology that could have utility for induction of specific immunologic nonreactivity to allografts, or other disease-associated antigens.

J. Clin. Invest. 109:755-764 (2002). DOI:10.1172/JCI200212142.

\section{Introduction}

There is considerable impetus to develop effective, clinically applicable methods to diminish the immunogenicity of alloantigens (1). Among the more promising approaches are those based on expression of donor alloantigens in recipient thymi. Intrathymic presentation of alloantigens can result in elimination or inactivation of potentially reactive $T$ cells by mimicking the natural processes that lead to negative selection of thymocytes bearing antigen receptors (TCRs) with high avidity for self-determinants (2). While several studies have shown that diverse allogeneic cells or peptides can efficiently induce specific tolerance after introduction into the thymi of recipients (3-7), these experimental methods may have limited feasibility or patient acceptance in clinical practice.

In an effort to develop alternative approaches, we explored the possibility that selected donor-derived cells may be able to traffic to thymi after intravenous injections. We were particularly interested in the homing properties of medullary thymic dendritic cells (TDCs), given the primary role of these cells in the processes of intrathymic negative selection $(8,9)$. While DC subpopulations isolated from spleen DCs (SDCs) or cultured from stem cells have been shown to have preferential trafficking to various regions of peripheral lymph nodes, and to immigrate poorly to thymi (10-13), the trafficking behavior of TDCs has not previously been detailed. Using adoptive transfer models in mice and sensitive assays, we have found that the thymic immigration of TDCs is several-fold greater than that of analogous SDC subpopulations after intravenous administration. Moreover, intravenous injections of relatively small numbers of TDCs were shown to result in central deletions of developing thymocytes and to induce nonreactivity to allografts. TDC intravenous treatment could have considerable advantages over other methods for intrathymic introduction of neoantigens, since it is a minimally invasive procedure and does not require prolonged, nonspecific immunosuppression. These novel findings may have substantial biologic significance by showing that disparate immunologic effects of DC subpopulations can be correlated with differential trafficking behaviors, and raise possibilities for the therapeutic use of cells that preferentially home to thymi and diminish specific $T$ cell responses.

\section{Methods}

Animals. Pathogen-free mice were obtained from the animal breeding facility at the Scripps Research Institute and littermates were randomly assigned to either experimental or control groups. Recipient animals were males of $\mathrm{H}-2^{\mathrm{b}}$ strains BALB.B, C57BL/6, or $2 \mathrm{C}$ TCR (14) (a gift of J. Sprent, The Scripps Research Institute), aged 4-12 weeks. DCs were harvested from $\left(\right.$ BALB.B $\times$ BALB/c) $F_{1}\left(H-2^{b / d}\right), B A L B / c\left(H-2^{d}\right)$, or $(\mathrm{C} 57 \mathrm{BL} / 6 \times \mathrm{SJL}) \mathrm{F}_{1}\left(\mathrm{H}-2^{\mathrm{b} / \mathrm{d}}\right)$ mice of either sex, varying in age from 4 weeks to 6 months. Organs for transplantations were harvested from male $\mathrm{F}_{1}$ mice (BALB.B $\times \mathrm{BALB} / \mathrm{c}$, or $\mathrm{C} 57 \mathrm{BL} / 6 \times \mathrm{SJL})$ and transplanted into male parental strain animals (e.g., BALB.B or C57BL/6, respectively). Thus, transplanted organs were highly immunogenic, being completely allogeneic at every MHC loci. Furthermore, transplants from $F_{1}$ animals into same-sex parental strains avoids confounding by minor histocompatibility $Y$ antigens or graft-versushost disease due to passive carriage of leukocytes. Protocols were approved by the Scripps Research Institute's Animal Research Committee. 
Cell preparations. Thymic or splenic DCs were obtained by collagenase digestion of pooled organs from euthanized donors, and the low-buoyant-density fraction was collected after centrifugation on Histopaque-1077 (Sigma-Aldrich, St. Louis, Missouri, USA). These cells were resuspended in RPMI supplemented with $10 \%$ FCS, $25 \mathrm{mM}$ HEPES, $2 \mathrm{mM}$ L-glutamine, $5 \times 10^{-5} \mathrm{M}$ 2-mercaptoethanol, penicillin, and streptomycin, and incubated in Petri dishes for 80 minutes at $37^{\circ} \mathrm{C}$ in $5 \%$ $\mathrm{CO}_{2}$. Nonadherent cells were removed by gentle washing with warm culture media, and the adherent cells collected after removal in $3.3 \mathrm{mM}$ EDTA with washes and gentle scraping (15). In some initial experiments, these thymic adherent cells (TACs) and splenic adherent cells (SACs) were used without further purification. In the majority of experiments, CD11 $c^{+}$cells were selected by FACS (Becton Dickinson and Co., San Jose, California, USA) from the adherent cell fractions, or, in later experiments, by treatment of low-buoyant-density cell fractions with anti-CD $11 c^{+}$-treated magnetic beads (CELLection; Dynal Inc., Lake Success, New York, USA) and detached using the manufacturer's protocol. DCs were resuspended in HBSS at $1.2 \times 10^{6}$ to $2.0 \times 10^{6}$ cells $/ \mathrm{ml}$ for injection into the tail veins of recipients.

Flow cytometry. FITC- or PE-conjugated mAb's, Annexin V, and 7-amino-actinomycin D (7-AAD) were obtained from PharMingen (San Diego, California, USA). We have found that DCs, particularly SDCs, nonspecifically bind streptavidin and antigen-presenting cells (data not shown). Nonspecific binding was minimized by use of Fc blocker (anti-CD16/32, PharMingen) and $2 \%$ syngeneic heat-inactivated mouse sera. Analyses were performed on 10,000 or more cells by FACScan II and analyzed with CellQuest software (Becton Dickinson and Co.) using gates for live cells (phenotypic characterizations) or for all cells (Annexin $\mathrm{V}$ and 7-AAD assays of thymocyte killing).

Preconditioning regimens. T cells were partially depleted from both experimental and control recipients prior to transplantation by treatments with 3 Gy total body irradiation, delivered by a ${ }^{137} \mathrm{Cs}$ source at $0.7 \mathrm{~Gy} / \mathrm{min}$, and a single intraperitoneal injection of $500 \mu \mathrm{g}$ of anti-CD3 $\mathrm{mAb}$ (145-2C11). Effects of these treatments on splenic $T$ cell populations were evaluated by multiplying the number of viable cells in splenocyte suspensions (determined by hemocytometer after lysis of red blood cells with hypotonic buffer) by the percentage of $\mathrm{CD}^{+}$cells in the suspensions (enumerated by flow cytometry).

Organ transplants. In order to assay for in vivo immunologic effects, we initially used a murine model for human lung transplantation consisting of heterotopic airway grafts (HAGs) (16-18). Tracheae and multiple generations of bronchi were obtained en bloc by blunt dissection and gentle teasing of lung parenchyma. After irrigation with RPMI, the airway preparations were implanted heterotopically in the interscapular subcutaneous tissues of methoxyfluraneanesthetized animals. Tail skin allografts were also performed on the lateral thorax of tribromoethanol- anesthetized mice using previously described methods (19). Transplants were performed 1 week after treatment with intravenous injections of cells or corresponding injections of HBSS (controls).

Rejection scoring. Syngeneic HAGs retain a normal histologic appearance indefinitely, as assessed by light microscopy. In contrast, allogeneic HAGs become firmly enveloped by a well-vascularized fascia within a few days. They subsequently undergo a succession of epithelial changes that replicate advancing stages of chronic rejection (obliterative bronchiolitis) in human pulmonary allografts, including progressive degrees of squamous metaplasia followed by denudation, and, in more advanced stages, increasing luminal obliteration by granulation tissue (16-18). Harvested grafts were fixed and stained with hematoxylin and eosin. While blinded to treatment, researchers assigned a score to each sample based on the most advanced histologic change present: 0 , normal; 1 , focal squamous metaplasia (in aggregate comprising less than $10 \%$ of luminal circumference); 2 , metaplasia comprising less than $10 \%$ of luminal circumference; 3 , focal denudation (in aggregate comprising less than $10 \%$ of luminal circumference); 4 , denudation comprising more than $10 \%$ of luminal circumference; and 5, intraluminal granulation tissue (obliterative bronchiolitis).

Skin transplants were assessed daily. They were scored as rejected when the graft was completely desiccated and/or scabbed (19).

Proliferation assays. Single-cell suspensions of recipient splenocytes $\left(4 \times 10^{5}\right)$ were cocultured with $4 \times 10^{5}$ irradiated (10 Gy) splenocytes from either syngeneic (control) or allogeneic strains in flat-bottomed 96-well culture plates $(200 \mu \mathrm{l} /$ well) for 3 days in an atmosphere of $5 \% \mathrm{CO}_{2}$ at $37^{\circ} \mathrm{C} .{ }^{3} \mathrm{H}$-thymidine was added $(1 \mu \mathrm{Ci} /$ well $)$, and cells were harvested 12 hours later. The stimulation index was calculated as mean cpm of allogeneic cultures/mean cpm of controls.

I-E $\alpha$ PCR. The design of this assay was based on the presence of an approximately 650-bp segment in the promoter and first exon of the $I-E \alpha$ gene in $\mathrm{BALB} / \mathrm{c}$ mice that is deleted in the genome of BALB.B animals (20). Genomic DNA was extracted by digestion with proteinase $\mathrm{K}$, and $200 \mathrm{ng}$ was subjected to $20-\mu \mathrm{l}$ PCR amplifications using Taq polymerase. Identical conditions were used in both reactions of the seminested PCR (30 cycles of $94^{\circ} \mathrm{C}$ for 30 seconds, $55^{\circ} \mathrm{C}$ for 30 seconds, and $72^{\circ} \mathrm{C}$ for 90 seconds). All primers corresponded to the segment uniquely present in BALB/c genomes. Five microliters of product of the first PCR, which used primers $I-E \alpha^{d}$ S2 (5'-GAGGTACAAATCCCCATTTTC-3') and $I-E \alpha^{d}$ AS3 (5'-TAACTGCCTG ATAACCTTCA-3'), was reamplified using $I-E \alpha^{d} A S 3$ and internal primer $I-E \alpha^{d} \mathrm{~S} 4$ (5'-TATTCT AGCCTCACTGATGT-3'). Ten microliters of this product was electrophoresed and stained with ethidium bromide. Validation studies using titrated mixtures of cells showed that the $I-E \alpha$ product was consistently evident in comixtures of $1 \times 10^{2}\left(\right.$ BALB.B $\times$ BALB/c) $F_{1}$ cells with $1 \times 10^{7}$ BALB.B splenocytes (data not shown). 
${ }^{111}$ In assays. DCs were radiolabeled using methods that have been described elsewhere $(10,11)$. Microautoradiographs were prepared using fixed and paraffin-embedded thymi harvested 2 days after intravenous injection of $5 \times 10^{5}{ }^{111} \mathrm{In}$-labeled DCs. After sectioning and hematoxylin and eosin staining, slides were dipped in Kodak Emulsion NTB-2 (VWR International, Brisbane, California, USA) diluted 1:1 with distilled water, air-dried, and then incubated in a lightsafe box at $4^{\circ} \mathrm{C}$. After 25 days of exposure, slides were immersed in Kodak D-19 developer, fixed in Kodak Rapid Fixer (VWR International), and examined using darkfield microscopy. ${ }^{111}$ In activity in tissues was determined by measuring cpm of weighed tissues, harvested 3 days after intravenous injection with ${ }^{111}$ In-labeled DCs, using an Atomlab 50 counter (Biodex Medical Systems, Shirley, New York, USA) calibrated for this isotope. Radioactivity was expressed as cpm/tissue weight and as a percentage of total radioactivity recovered/tissue weight (specific activity), thus allowing for differences in size of tissue and accessible label (11).

TCR profiles. Details of the methodology for measurement of TCR $\beta$ chain variable region (BV) gene expression have been previously reported (21). Total cellular RNA for these assays was obtained from splenocytes of euthanized animals. Individual TCR genes were quantitated as percentages of total BV expression.

Data analysis. Multiple group analyses of ordered data (histology scores) were performed using the KruskalWallis test. Parametrically distributed continuous values (proliferation indices) were evaluated by ANOVA. Subsequent multiple comparisons were made using the Newman-Keuls test. Comparisons of skin graft survival durations were made by the Kaplan-Meier product-limit method, with analysis by log rank test. Significance was defined as $P<0.05$. Data are shown as mean \pm SEM.

\section{Results}

Injections of donor TDCs protect airway allografts. Initial experiments showed that intravenous injections of unsorted TACs from fully allogeneic donors, in conjunction with the preconditioning regimen, could result in protection of HAGs from rejection-associated histologic changes after transplantation. However, the number of cells necessary to achieve graft protection for at least 6 weeks was substantial $\left(1 \times 10^{7}\right.$ to $2 \times 10^{7}$ per recipient), and smaller inoculations did not exert an apparent beneficial effect. Injections of unsorted donor SACs in similar or greater numbers were associated with exacerbated destruction of allografts (data not shown).

The preconditioning regimen (described in Methods) was used immediately prior to intravenous injections of DCs (or other cells) in these and subsequent experiments. The rationale for this treatment derives from prior experiments using direct intrathymic injections of antigenic cells or peptides (3-7), and appears necessary in order to decrease the precursor frequency of periph- eral, alloreactive T cells that are already present. A small number of experiments conducted without preconditioning confirmed the requirement for this treatment. This preconditioning regimen is only transiently immunosuppressive, however, resulting in a reduction of splenic $\mathrm{CD}^{+}$cells from $1.9 \times 10^{7} \pm 0.5 \times 10^{7}$ to $6.1 \times$ $10^{5} \pm 0.1 \times 10^{5}$ cells/spleen in recipients 3 days after treatment $(n=6)$, whereas normal numbers of splenic $\mathrm{CD}^{+}$cells $\left(2.1 \times 10^{7} \pm 0.3 \times 10^{7}\right)$ were seen by day 10 .

We hypothesized that the cell type most responsible for effects of intravenous TAC injections was likely to be the medullary TDCs. These cells, defined and isolated by their expression of CD11c $(8,14,22,23)$, constituted $1-3 \%$ of the highly heterogeneous TACs. Other phenotypic characteristics were consistent with those reported for TDCs, including morphology in culture and localization in immunohistochemically stained sections of thymi (data not shown). CD8 $\alpha(22-24)$ was present on more than $85 \%$ of the TDCs (Figure 1). In contrast with TDCs, a minority of the SDCs (less than 20\%) expressed CD8 $\alpha(14,22$, 23). Both TDCs and SDCs expressed high levels of MHC class I and II, and had similar levels of costimulatory molecules (Figure 1). FasL was expressed at low levels by TDCs and SDCs $(3.3 \% \pm 0.6 \%$ and $5.9 \% \pm 1.2 \%$, respectively). As previously reported (22), there was considerably greater surface expression of FasL among SDCs bearing the proposed lymphoid marker CD8 $\alpha$ (S-LDCs) than among the putative myeloid-derived splenic DCs (S-MDCs) $(21.7 \% \pm 4.9 \%$ vs. $5.1 \% \pm 2.8 \%$, respectively).

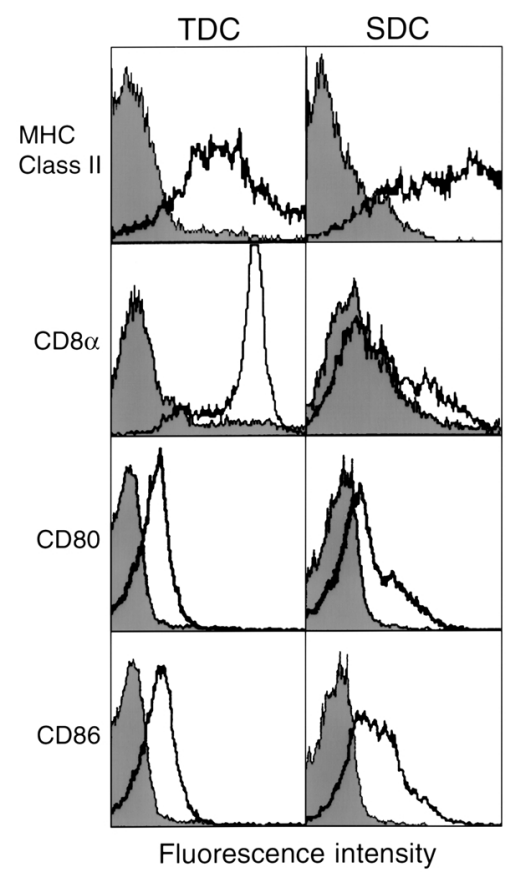

Figure 1

Phenotypes of TDCs and SDCs. Frequencies versus $\log _{10}$ fluorescence intensities of the markers are depicted by the white profile. The gray profile indicates background staining. Results shown are representative of 6 or more experiments with similar results. 


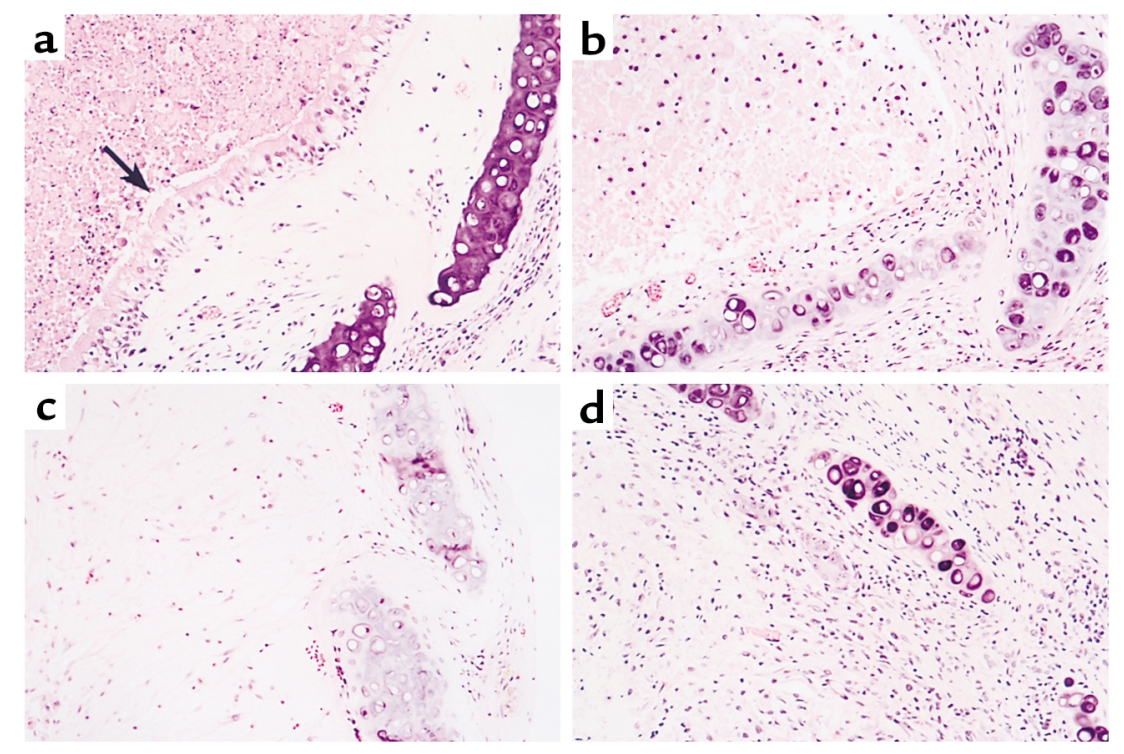

\begin{abstract}
Figure 2
Histologic evaluations of HAGs. (a) Hematoxylin and eosin-stained section of a typical (BALB.B $\times$ BALB/c) $F_{1}$ allograft harvested 6 weeks after implantation in a TDC-treated $\left(1.5 \times 10^{5}\right.$ intravenous TDCs) BALB.B recipient. The mucin-filled airway lumen is denoted by an arrow, which points to viable epithelial mucosa. HAGs harvested from (b) Allogenic HAGs from recipients treated with donor-derived CD11 $c^{-}$thymic adherent cells (other TAC) show greater degrees of allograft rejection with squamous metaplasia and denudation. Allografts harvested from animals treated with $(\mathbf{c})$ saline injections (controls) and (d) intravenous SDCs $\left(1.5 \times 10^{5}\right.$ cells) show epithelial destruction and airway obliteration. No beneficial effects of intravenous SDC treatments were seen at any dose tested (ranging up to $2.0 \times 10^{6}$ SDCs).
\end{abstract}

Treatments with $1.2 \times 10^{5}$ to $2.0 \times 10^{5}$ TDCs from $($ BALB.B $\times$ BALB $/ \mathrm{c}) \mathrm{F}_{1}$ donors injected into tail veins of BALB.B recipients, in conjunction with preconditioning, resulted in protection of donor-strain HAG from the epithelial injuries of allograft rejection for up to 8 weeks (Figure 2a). Allografts harvested from animals treated with $1.2 \times 10^{5}$ to $2.0 \times 10^{5}$ intravenous CD11 $\mathrm{c}^{-}$ TACs showed changes consistent with moderate to severe rejection (Figure 2b). HAGs from control animals that received an identical preconditioning regimen, but no cell injections, typically showed severe rejection responses (Figure $2 c$ ). Treatments with equal or up to tenfold greater numbers of donor SDCs also resulted in severe rejection (Figure 2d). Quantitative analyses of histopathology scores further illustrated the relative difference between animals receiving these various treatments (Figure 3a).

Graft survival prolongation by intravenous TDCs was dose-dependent (requiring $>1.0 \times 10^{5}$ ). TDCs from fully allogeneic $\mathrm{BALB} / \mathrm{c}$ mice were equally effective as $\left(\right.$ BALB.B $\times$ BALB/c) $F_{1}$ TDCs in protecting $\mathrm{BALB} / \mathrm{c}$ HAGs transplanted into BALB.B recipients. Treatment effects were strain-specific, however, in that (BALB.B $\times$ $\mathrm{BALB} / \mathrm{c}) \mathrm{F}_{1}$ TDCs did not protect $(\mathrm{B} 6 \times \mathrm{SJL}) \mathrm{F}_{1}$ HAGs from developing severe rejection after transplantation into C57BL/ 6 recipients $(n=10)$. The thymic dependence of these effects was confirmed by experiments in which 6-week-old HAGs harvested from animals that had been thymectomized 2 weeks prior to preconditioning and intravenous TDC injections showed severe rejection (indistinguishable from Figure $2 c, n=7$ ). Analyses of lymphocyte subsets in the thymectomized animals showed that they had undergone considerable expansions of $\mathrm{T}$ cells in the interval since preconditioning, probably due to homeostatic (25) and/or alloantigen-driven (21) proliferation. The thymectomized animals had ratios of $\mathrm{CD}^{+} / \mathrm{CD} 5^{+}(0.11 \pm 0.01)$ and $\mathrm{CD}^{+} / \mathrm{CD}^{2} 5^{+}(0.05 \pm 0.01)$ that were approximately onehalf those of control (previously preconditioned, thymic intact) animals $(0.23 \pm 0.02$ and $0.07 \pm 0.01$, respectively). Thus, the numbers of potentially alloreactive $T$ cells in the thymectomized animals is predictably more than adequate to mount substantive responses $(21,26)$. Skin allograft survival is prolonged by TDCs. In order to further evaluate in vivo effects of intravenous TDCs under especially stringent conditions, we performed skin transplants from $(\mathrm{B} 6 \times \mathrm{SJL}) \mathrm{F}_{1}$ mice onto preconditioned $\mathrm{C} 57 \mathrm{BL} / 6$ mice. The use of this alternative donor-recipient strain combination also enabled us to ascertain that treatment effects of TDCs were not unique to the BALB/c $\rightarrow$ BALB.B combination. Mean allograft survival was prolonged more than $70 \%$, compared with preconditioned controls, by a single intravenous injection of $1.2 \times 10^{5}$ to $2.0 \times 10^{5}$ TDCs (from $20 \pm 2$ days to $35 \pm 2$ days) (Figure 3b). Skin allograft survival was not prolonged relative to controls by similar treatments with the same number of intravenous SDCs $(19 \pm 3$ days, $n=3)$ or by comparable treatments with SDC subpopulations that had been segregated into $\mathrm{CD} 8 \alpha^{-}(24 \pm 1$ days, $n=4)$ or $\mathrm{CD} 8 \alpha^{+}(20 \pm 2$ days, $n=3$ ) fractions (S-MDCs and S-LDCs, respectively).

In vitro evidence of specific immunologic hyporesponsiveness. Effects of TDC injections were further corroborated by results of one-way mixed lymphocyte reactions, wherein 
splenocytes from treated animals were cocultured with irradiated donor strain splenocytes. While splenocytes from TDC-treated animals did not proliferate when cultured with donor strain cells (stimulation index $=$ $1.1 \pm 0.2$ ), values were approximately fourfold greater in mixed lymphocyte reactions of recipients treated with SDCs and in control animals $(P<0.01)$ (Figure $3 c)$. As was seen with in vivo HAG assessments, treatments of C57BL/6 animals with TDCs from (BALB.B $\times$ $\mathrm{BALB} / \mathrm{c}) \mathrm{F}_{1}$ mice did not ameliorate mixed lymphocyte reaction responses against third-party $(\mathrm{SJL})$ cells (stimulation index $=3.68 \pm 0.4, n=5$ ).

In vitro stimulation of T cells by $D C$. To exclude the possibility that the prolongation of allograft survival with TDC injections was due to a direct suppressive or veto action of these cells (22-24), we compared the abilities of DC subpopulations to stimulate naive allogeneic immune responders by measuring ${ }^{3} \mathrm{H}$-thymidine incorporation. These experiments were designed to simulate in vivo conditions with intravenous injections of DCs, i.e., relatively small numbers of DCs were transferred into animals with much larger numbers of admixed allogeneic immune effectors. As depicted in Figure 3d, the responses evoked by allogeneic TDCs in these mixed cultures were dose-related and comparable to those induced by S-MDCs, whereas S-LDCs had a significantly lesser stimulant effect on proliferation.

Cell homing. We speculated that the unique ability of TDCs to increase allograft survival with intravenous injection may be due to a greater relative capability of these cells to immigrate to thymi. In order to test this hypothesis, sensitive assays were devised to evaluate in vivo trafficking behavior. Given the small numbers of injected TDCs and the presence of many native thymic cells with autofluorescence and/or enhanced nonspecific binding, initial efforts using more conventional methods of cell tracking (mAb's or intracellular dyes) generally resulted in unfavorable signal-to-noise ratios. PCR of genomic DNA extracted from thymi of BALB.B animals treated 1 week previously with $2 \times 10^{5}$ (BALB.B $\times \mathrm{BALB} / \mathrm{c}) \mathrm{F}_{1}$ TDCs showed the presence of I-E $\alpha$ products in all animals tested $(n=6)$ (Figure $4 a)$. In contrast, PCR after similar treatments with identical numbers of SDCs consistently failed to produce the BALB/C I-E $\alpha$ band $(n=4)$. Injections of tenfold greater numbers of SDCs $\left(2 \times 10^{6}\right.$ cells/recipient $)$ yielded BALB $/ c I-E \alpha$ gene
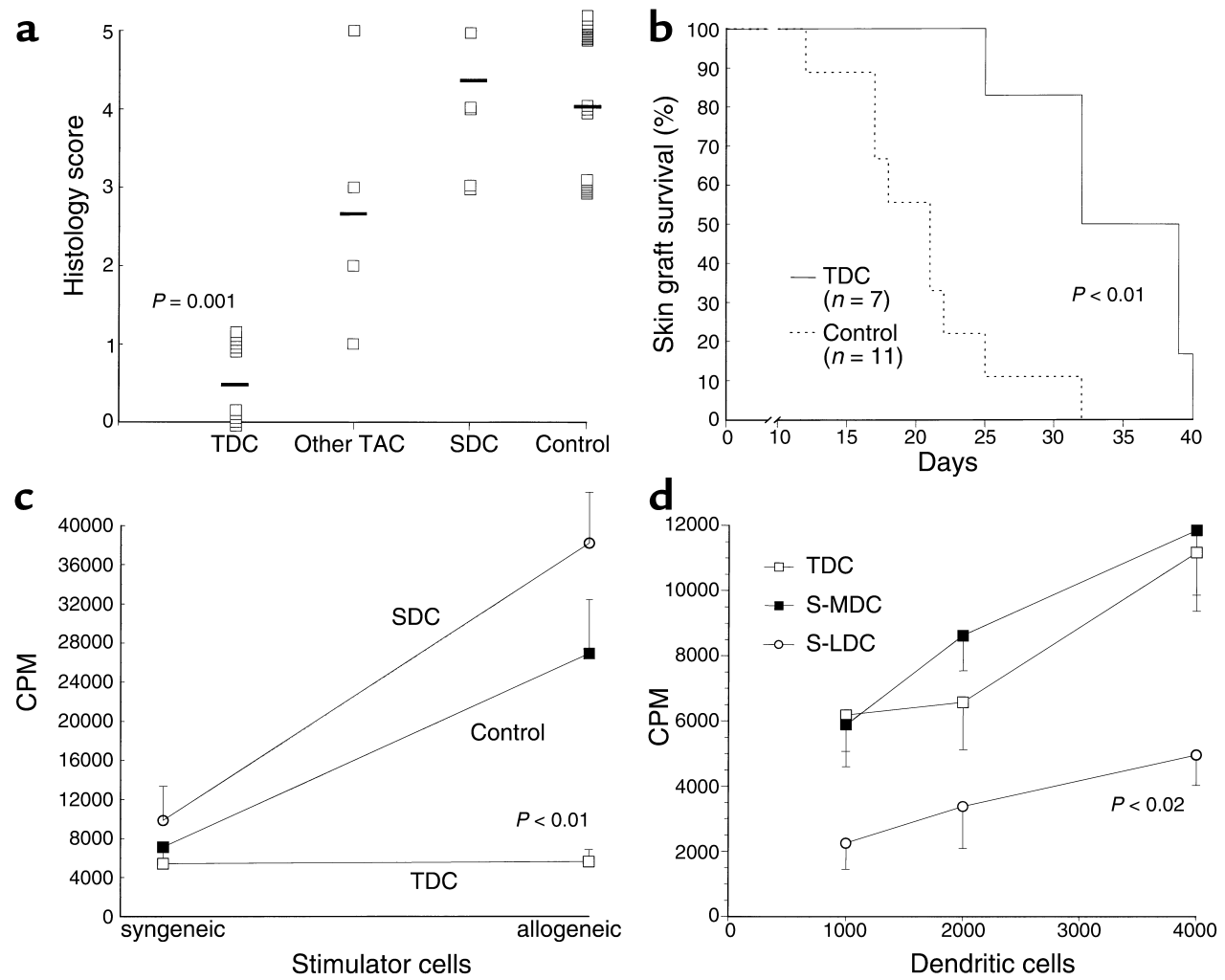

Figure 3

(a) Histology scores of airway allografts harvested 6 weeks after transplantation using the donor/recipient strain combinations described in Figure 2. Mean values are depicted by horizontal bars. TDC scores were significantly lower than those of other groups. (b) Skin allograft survival is prolonged by intravenous injections of TDCs. In these experiments, $(B 6 \times S J L) F_{1}$ tail skin was grafted onto $B 6$ recipients $(\mathbf{c})$. ${ }^{3} \mathrm{H}$-thymidine incorporation of HAG recipient (BALB.B) splenocytes cocultured with irradiated allogeneic BALB/c donor cells or syngeneic BALB.B controls (pooled data of replicate trials). No proliferation was observed in animals treated with TDCs $(n=7)$, in contrast with values of animals treated with SDCs $(n=6)$ and values of control animals (no cell injections) $(n=8)$. (d) Stimulatory effect of BALB/c DCs in cocultures with naive allogeneic (BALB.B) splenocytes. Results shown are ${ }^{3} \mathrm{H}$-thymidine cpm of cocultures minus baseline cpm (no allogeneic DCs), with results pooled from replicate trials ( $n=13$ in each DC group). 


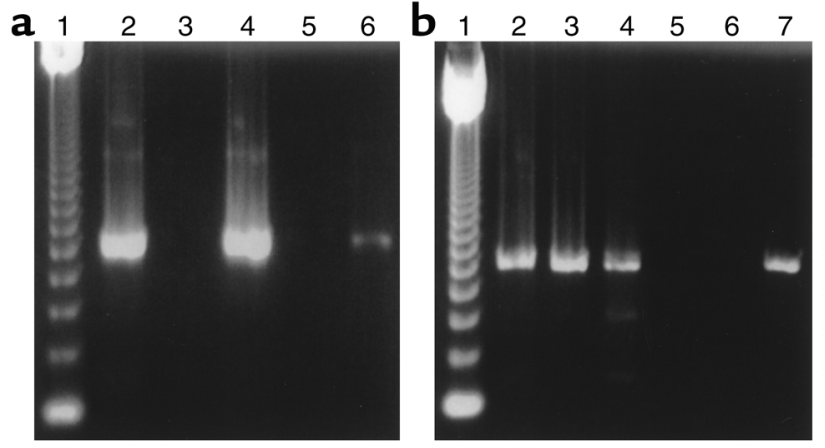

Figure 4

PCR evaluation of cell migration. Lane 1 of both figures shows the 123-bp marker. (a) PCR of genomic DNA extracted from thymi after injections of DCs. Lane 2 shows positive BALB/c l-E $\alpha$ product in thymus of BALB.B recipient 1 week after injection with $2 \times 10^{5}$ (BALB. $B$ $\times B A L B / c) F_{1}$ TDCs. Lane 3 depicts the absence of product in an animal injected with $2 \times 10^{5}$ of SDCs. Lane 4 shows PCR of thymus DNA extracted from animal injected with $2 \times 10^{6}$ SDCs. Lane 5 is a negative (reagents only) control, and lane 6 is a positive control consisting of DNA extracted from a mixture of $1 \times 10^{3}($ BALB.B $\times B A L B / c) F_{1}$ splenocytes and $1 \times 10^{7}$ BALB.B splenocytes. Lanes 4 and 5 show results 4 weeks after injection of $2 \times 10^{6} \mathrm{SDCs}$ (only one of eight treated animals was positive). (b) Persistence of allogeneic DCs in thymi after intravenous injections. Lanes 2 and 3 show I-E $\alpha$ products in thymi of recipients injected 4 weeks earlier with $2 \times 10^{5}$ TDCs. Lanes 4 and 5 show results 4 weeks after injection of $2 \times 10^{6}$ SDCs (only one of four treated animals was positive). Lanes 6 and 7 are negative and positive controls, respectively, as described in $\mathbf{a}$.

products that were qualitatively similar to results with smaller numbers of TDCs (Figure 4a).

In order to examine the persistence of thymic chimerism after intravenous injections with allogeneic DCs, we performed PCR analyses of thymi from BALB.B animals at various timepoints after injection with either $2 \times 10^{5}$ TDCs or $2 \times 10^{6}$ SDCs derived from BALB/c animals. Following TDC injections, products of the $\mathrm{BALB} / \mathrm{c}$ gene were detected in all thymi from animals injected either 1 week $(n=4)$ or 2 weeks $(n=4)$ earlier, and in seven of eight animals 4 weeks after injection (albeit with progressively diminishing band intensities as a function of elapsed time). While chimerism, based on the presence of faint $\mathrm{BALB} / \mathrm{c}$ PCR products, was evident in all SDC-treated animals 1 week $(n=4)$ and 2 weeks $(n=4)$ after injection, BALB/c I-E $\alpha$ DNA could be detected in only one of eight animals at 4 weeks after injection (Figure $4 \mathrm{~b}$ ). None of the PCRs performed on thymi of animals 6 weeks after injection of either TDCs or SDCs were positive ( $n=6$ for each group).

To further and independently evaluate the thymic homing behavior of DCs, microautoradiographs of thymic sections were developed from animals that had been injected with ${ }^{111}$ In-labeled cells. Specimens from animals injected with radiolabeled TDCs showed comparatively high activity in proximity to thymic perivascular areas, whereas only rare, isolated foci of radioactivity could be detected in animals receiving SDCs (Figure 5, a and b).
Quantitative determinations of radioactivity were also performed in organs harvested from animals after injection with ${ }^{111} I n$-labeled DCs. Tissue distribution analyses of radiolabeled TDCs and SDCs showed markedly greater thymic homing of the former, whereas splenic homing was enhanced among the latter (Figure 5c). Subsequent experiments comparing TDC to SDC subpopulations, segregated by CD8 $\alpha$ expression, showed no appreciable variances in organ distribution between the two SDC groups, and thymic homing of TDCs was much greater than that of both splenic cell types, as evidenced by several measures, including specific activity (Figure 5d) and absolute $\mathrm{cpm} / \mathrm{g}$ thymus tissue $(3,601 \pm 614$ vs. $294 \pm 113$ and $411 \pm 161 \mathrm{cpm} / \mathrm{gm}$; TDCs, S-MDCs, and S-LDCs, respectively, $P<<0.01$ ).

Thymocyte deletions. Given that induction of allogeneic hyporesponsiveness caused by treatment with intravenous TDCs appeared to be a thymic mechanism, we
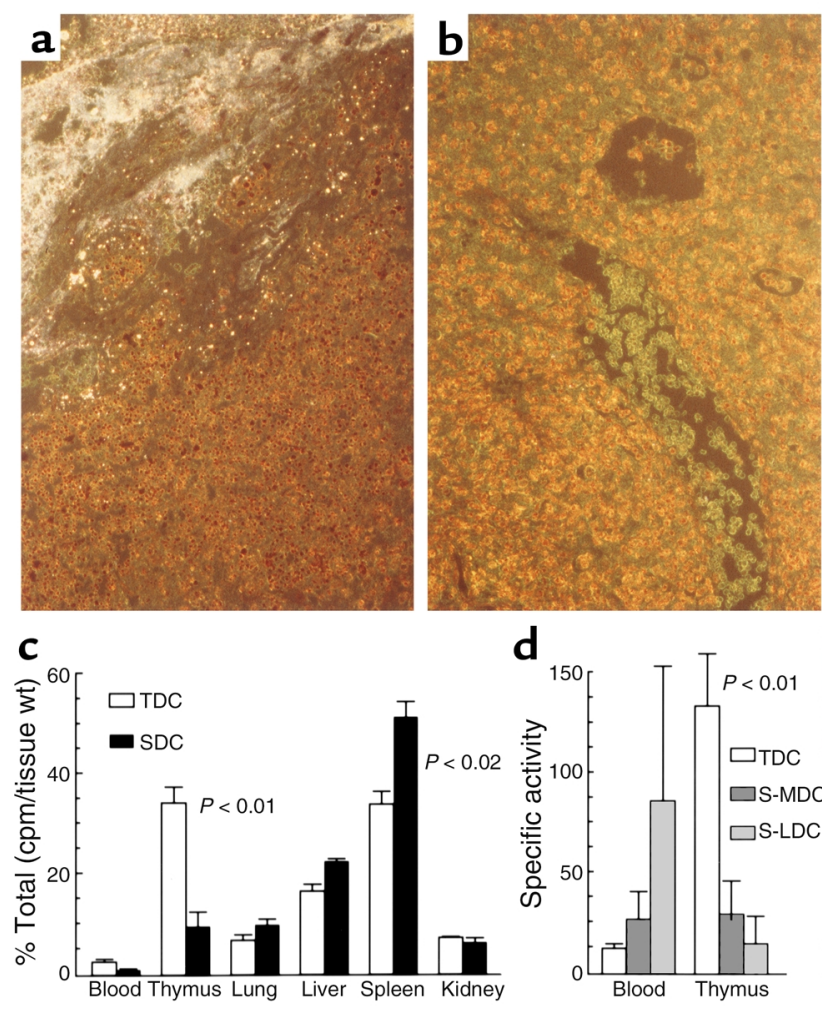

\section{Figure 5}

Microautoradiographs of thymi harvested from BALB.B mice injected intravenously with ${ }^{111} / n$-labeled BALB/c TDCs (a) and SDCs (b). Light areas correspond to radioactivity of labeled DCs in perivascular spaces. (c) Three days after injection with ${ }^{111} / n$-labeled DCs $(n=3)$, $\mathrm{cpm} /$ tissue weight of various organs harvested from animals was determined (expressed here as a percentage of total). (d) Specific activity (see Methods) of organs harvested from animals after injection with ${ }^{111} / n$-labeled DCs (pooled results of three trials using more than four animals/group in each trial). Activity in blood was appreciably greater among the SDC populations and approximated or exceeded that found in thymi. In contrast, specific activity among thymi of TDC-injected animals was much greater than that of SDCinjected animals and was more than 11-fold that of concurrent blood specimens (consistent with preferential intrathymic localization). 
sought direct evidence that these cells exert effects by causing central deletions of alloreactive thymocytes. TCR repertoires of $B A L B / c$ mice are characterized by intrathymic deletions of BV3, BV5, BV11, and BV12, due to superantigenic effects of endogenous mouse mammary tumor proviruses, whereas BALB.B animals are unaffected since they do not express I-E (27). Accordingly, the intrathymic presence of cells expressing $I-E^{d}$ could result in reductions of these BVs in BALB.B animals, if the cells are also capable of participation in negative selection. This hypothesis was confirmed by evaluations of TCR peripheral repertoires in $($ BALB.B $\times$ BALB $/ c) F_{1}$ mice that showed reductions in these particular BV families compared with BALB.B mice, with the greatest reductions noted in BV3 $(0.24 \%$ $\pm 0.09 \%$ vs. $1.08 \% \pm 0.08 \%$ of total BV).

We then evaluated the ability of injected TDCs to alter TCR repertoires using this model system. Although DCs may mediate superantigen-induced deletions relatively inefficiently (28-31), and the incomplete depletion of the preconditioning leaves many peripheral $T$ cells (with their original TCR BV) unaffected, we nonetheless found that BALB.B mice treated 6 weeks earlier with preconditioning and intravenous BALB/c TDCs $(n=6)$ exhibited reductions of these $\mathrm{BV}$ relative to controls $(n=11)$. The most evident and consistent reductions were again noted among BV3 $(0.72 \% \pm 0.13 \%$ vs. $1.08 \% \pm 0.08 \%$ of total $\mathrm{BV}, P<0.03)$. In contrast, no appreciable BV3 reductions could be discerned in analyses of cells from animals $(n=4)$ that had been intravenously injected with SDCs $(1.21 \% \pm 0.1 \%$ of total BV).

While the BV analyses showed that intravenous TDCs can shape the peripheral TCR repertoire of recipients, we sought further evidence that the deletions were occurring in thymi. For this purpose, we assayed death of double positive $\left(\mathrm{CD}^{+} \mathrm{CD}^{+}\right)$thymocytes by flow cytometry after intravenous injections of $($ BALB.B $\times \mathrm{BALB} / \mathrm{c}) \mathrm{F}_{1}$ TDCs or SDCs into $(\mathrm{C} 57 \mathrm{BL} / 6 \times 2 \mathrm{C}$ TCR $) \mathrm{F}_{1}$ mice that were otherwise untreated (no preconditioning). Approximately $20 \%$ of peripheral $\mathrm{T}$ cells in these animals bear a transgenic TCR with high avidity against the BALB/c MHC Class I $L^{d}(14)$. Effects were measured by flow cytometric evaluations: of apoptosis using Annexin $V$ and of cell death by measuring uptake of 7-AAD. Results of both measurements were closely correlated in individual animals $(r=0.8, P<0.01)$. Negative selection of immature thymocytes was much greater among TDC-treated animals than in either the SDC-injected group or noninjected littermate controls (Figure 6).

To further test whether SDCs were inherently capable of inducing central deletions (had they been able to home to thymi in adequate numbers), we injected $1 \times 10^{5} \mathrm{BALB} / \mathrm{c}$ SDCs directly into thymi of $2 \mathrm{C}$ TCR transgenic animals. The extent of thymocyte death following direct intrathymic injection of these cells was comparable to that caused by intravenous injections of TDCs (Figure 6). These effects were due to deletions caused by the allogeneic cells, since similar treatments with syngeneic SDCs did not cause measurable increases in thymocyte apoptosis compared with uninjected controls.

\section{Discussion}

These data show that TDCs were able to immigrate to thymi of allogeneic recipients after intravenous injections, where they caused deletions of immature thymocytes and TCR repertoire alterations. Moreover, single injections of relatively small numbers of TDCs resulted in prolonged survival of donor-strain grafts, as well as specific in vitro unresponsiveness, in stringent models with donor-recipient MHC mismatches at every locus. In contrast, SDCs exhibited relatively poor thymic homing after intravenous injections, and these treatments did not appear to diminish alloreactivity. The present findings are a novel demonstration of markedly differential immunologic effects among DC subpopulations due
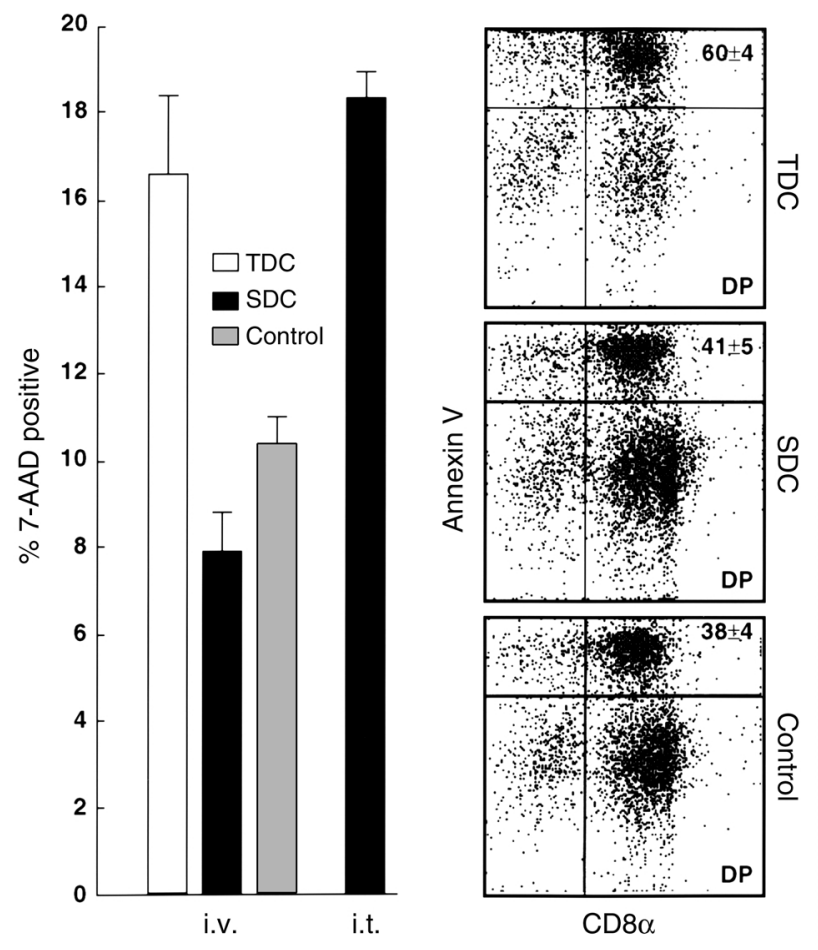

\section{Figure 6}

Thymocyte death in $(\mathrm{C} 57 \mathrm{BL} / 6 \times 2 \mathrm{CTCR}) \mathrm{F}_{1}$ animals injected with $\mathrm{BALB} / \mathrm{c}$ DCs. Left panel shows percentages of CD4 ${ }^{+} \mathrm{CD} 8^{+}$double positive thymocytes that stained with 7-AAD in animals treated with DCs administered by either intravenous or direct intrathymic injections. Thymocyte death was much greater with intravenous injections ofTDCs $(n=9)$ than with SDCs $(n=9)$ or control treatment $(n=3)$. Direct intrathymic injection of SDCs shows that these cells readily induce death of double positive thymocytes if they have access to the thymic microenvironment $(n=2)$. The right panel shows dot plots of thymocytes gated for CD4 expression. The percentages of Annexin $\mathrm{V}$-positive cells (undergoing apoptosis) are much greater among double positive thymocytes from intravenous TDC-treated animals than among those that received intravenous SDCs or control treatment. DP, double positive. 
to their divergent trafficking behaviors, and raise the possibility that thymus-homing cells could eventually be used to selectively induce hyporesponsiveness.

Differential in vitro immunologic activities of various DC subpopulations resulting from inherent, phenotypically determined properties have been extensively described, but the in vivo significance of these observations remains uncertain. As shown here and elsewhere, S-LDCs, which share at least some phenotypic similarities to TDCs, are less potent in vitro stimulants of $\mathrm{T}$ cell proliferation than are S-MDCs. The lesser stimulatory effect of S-LDCs has been attributed to their enhanced expression of FasL, which results in increased $\mathrm{CD}^{+} \mathrm{T}$ cell apoptosis (22). S-LDCs have also been reported to evoke Th2 responses in direct cocultures with naive $\mathrm{T}$ cells, whereas S-MDCs appeared to preferentially prime for Th1 differentiation (32). Directly contrary results, however, have been described with in vivo models $(24,33,34)$. Furthermore, S-LDCs have been shown to efficiently prime antigen-specific $\mathrm{T}$ cell responses in animals $(24,35)$, and as demonstrated here, do not prolong allograft survival after adoptive transfer. These seemingly disparate observations might be explained by recent findings that suggest that the Th responses elicited by DCs vary depending on the cellular microenvironment and the extent of their maturation, rather than being a fixed property determined by lineage $(34,36)$. Indeed, the notion that DC phenotypes (and functional effects) are directly determined by specific lineages has been disputed by recent evidence that both $\mathrm{CD} 8 \alpha^{+}$and $\mathrm{CD} 8 \alpha^{-}$subpopulations may be derived from common stem cells (37).

Neither the present data nor any report of which we are aware provides substantive evidence that TDCs have unique functions that can explain their generation of immunologic hyporesponsiveness by other than thymic mechanisms. As shown here and elsewhere (reviewed in ref. 8), TDCs have low-level FasL expression, and evoke strong proliferative responses in naive $T$ cells that equal or exceed those of S-MDCs, although these two cell types obviously have very dissimilar effects on allograft survival after intravenous injection. The ability of TDCs to spontaneously immigrate to thymi was shown here by multiple independent measures, and to our knowledge, has not been previously directly observed. These same methods also demonstrated that SDCs home comparatively poorly to thymi, and this particular finding has been corroborated by earlier studies (10-13). Support for the present data is also implied by a report that $4 \times 10^{6}$ or more syngeneic TACs that had been pulsed with myelin basic protein (MBP) peptides decreased the incidence of experimental allergic encephalo-myelitis after intravenous injections into Lewis rats, and no treatment effects were evident with administration of MBPpulsed SACs (38). While no data were presented that specifically demonstrated thymic homing, these investigators also found that prior thymectomy ablated effects of the TACs.
The relatively poor thymic homing of SDCs is undoubtedly a major determinant of their inability to obviate allograft reactivity. As predicted by models of thymocyte selection mechanisms (2), the presentation of antigens (including allogeneic peptides) in thymi by artificial delivery can result in apoptosis of maturing thymocytes bearing TCRs with sufficient avidities against these antigens. Direct thymic inoculations of a wide variety of antigen-presenting cells, including SDCs, have been shown to promote central deletions (as shown here), as well as induce allotolerance (3-7). Comparable and corroborative findings are provided by in vitro demonstrations that splenic SDCs (or other extrathymic antigen-presenting cells) cause deletions of reactive, immature T cells and specific allogeneic tolerance in thymic organ cultures $(14,39,40)$. Thus, any number of cell types that can present antigens, including SDCs, are inherently capable of inducing central negative selection and abrogating specific immune responses if they can gain access to the thymic microenvironment in adequate numbers.

Nonetheless, the reason or reasons for the failure of SDCs to induce some measure of allograft hyporesponsiveness after administration of very large numbers of these cells is uncertain. The sensitive measurements used here showed evidence for some thymic localization of SDCs with comparatively very large inocula. Nonetheless, repeated efforts to induce allohyporesponsiveness with intravenous injections of high-dose SDCs (or bone marrow DC cultures, data not shown) have been unsuccessful. Multiple analogous reports have also shown that even larger numbers of injected, unmodified SDCs (or DCs cultured and expanded in vitro) fail to decrease immunologic reactivity $(13,38,41$, 42). In fact, the adoptive transfer of splenic and/or cultured DCs has shown considerable promise for therapeutic immunizations (43). The failure of injected SDCs to prolong graft survival here may be due to an inability to migrate from the vascular compartment (even if adherent to thymic endothelium) into the medullary regions where negative selection occurs. Alternatively, even if some SDCs do enter thymi after massive inoculations, whatever propensity for down regulating responses may ensue could be substantially outweighed by the presence of very large numbers of these potent immunogens in peripheral lymphoid organs. The PCR data from this study also indicates that thymic persistence of allogeneic SDCs, likely due to early cell demise or subsequent remigration, is much less than that of TDCs. It is tempting to speculate that discrete mechanisms might exist to exclude peripheral DCs from the thymic medullary compartment as a way to ensure the maintenance of immunologic responses to acquired non-self antigens that are processed and presented by these cells in spleen and lymph nodes.

Central deletions of alloreactive precursors are likely a major mechanism for the induction of allogeneic hyporesponsiveness by intravenous TDCs, as shown by effects of these injections on TCR repertoires and 
thymocyte apoptosis. Similar conclusions have been drawn by others in their analogous studies using direct intrathymic injections of donor-derived cells or peptide antigens $(3,4,44)$, DC admixtures in thymic organ cultures $(14,39)$, or intrathymic expression of antigen in TCR transgenic systems (45). Nonetheless, the clonal deletion process is imperfect, even with lifelong, constitutively high levels of intrathymic antigen expression, and some potentially reactive thymocytes escape deletion and are exported to the periphery (46, 47). Typically, however, these $T$ cells have been found to be relatively (or completely) hyporesponsive to the specific intrathymic antigens, an effect that has been variously attributed to induction of thymus-dependent anergy $(44,47)$, generation of regulatory lymphocytes (48), or a repertoire limited to low-avidity TCRs that require very high levels of antigen (46). Comparable results are implied by the present findings that the potentially alloreactive $T$ cells that undoubtedly escaped deletion during the first few weeks after TDC treatments did not proliferate in vitro when cocultured with donor cells, whereas these animals retained reactivity to third-party cells and allografts.

The eventual ability to induce specific immunologic hyporeactivity by injections of thymic homing cells could ultimately offer several substantial advantages over existing methods. While the functional capabilities of thymi are retained well into maturity $(49,50)$, these organs undergo involution after puberty and eventually become isolated and dispersed islets in the anterior mediastinum. Thus, therapies requiring direct intrathymic injections of alloantigens or cells in older recipients are likely to require invasive exposures and/or extensive injections. Moreover, since the life span of injected donor cells is finite, effective treatment in the clinical setting would probably involve multiple courses. Colonization of recipient thymi with donor leukocytes by establishment of bone-marrow chimeras has also been used for induction of experimental graft tolerance. However, inherent difficulties in these approaches currently preclude use in human transplant recipients, including problems establishing and maintaining engraftment, especially across mismatched human leukocyte antigens, as well as risks of graft-versus-host disease that would necessitate protracted nonspecific immunosuppression $(1,51)$. Aside from an initial, transient $\mathrm{T}$ cell depletion, however, immune modulation using donor-derived thymic homing cells would be highly directed against specific antigen-reactive thymocytes, and other cellular immune responses would remain fully intact.

In summary, the present data are a demonstration that distinctly differential trafficking behavior is an important difference among DC subpopulations that mediate immunologic functions. The findings here that adoptively transferred $\mathrm{CD} 8 \alpha^{+}$DCs from thymus and spleen have markedly divergent biologic effects, despite sharing markers previously thought to denote a common ontogeny, is further evidence that the immune activities of particular DC subpopulations cannot necessarily be characterized on the basis of simple phenotypes. These findings are consistent with and extend an emerging view that the particular environment (and by extension, the trafficking behavior) of a given DC subpopulation plays a critical role in determining the immunologic properties of these cells (36, 37). The potential therapeutic applicability of thymushoming DCs will predictably be advanced by the eventual ability to propagate (52) or immortalize (53) these cells, or to isolate thymus-homing stem cells (37). We further anticipate that the duration of beneficial effects will likely be optimized by upward titration of TDC doses, serial administrations, and/or manipulations that could prolong the life span of these cells $(53,54)$. With further development, intravenous administration of thymus-homing cells could be a minimally invasive method for the induction of antigen-specific hyporesponsiveness to allografts, and analogous treatments with peptide-pulsed cells could potentially ameliorate pathologic $\mathrm{T}$ cell responses to self-antigens.

\section{Acknowledgments}

The authors thank Jonathan Sprent, Wendy Havran, Nicholas Gascoigne, and Jonathan Kaye for their critical reviews of this manuscript. This work was supported in part by NIH grants 1KO8 HL-03016, 1RO1 HL-64192, and AR-31203.

1. Turka, L.A. 1998. What's new in transplant immunology: problems and prospects. Ann. Intern. Med. 128:946-948.

2. Ashton-Rickardt, P.G., and Tonegawa, S. 1994. A differential-avidity model of T-cell selection. Immunol. Today. 15:362-366.

3. Markmann, J.F., et al. 1993. Deletion of donor-reactive T lymphocytes in adult mice after intrathymic inoculation with lymphoid cells. Transplantation. 55:871-877.

4. Naji, A. 1996. Induction of tolerance by intrathymic inoculation of alloantigen. Curr. Opin. Immunol. 8:704-709.

5. Sayegh, M.H., et al. 1993. Thymic recognition of class II major histocompatibility complex allopeptides induces donor-specific unresponsiveness to renal allografts. Transplantation. 56:461-465.

6. Dono, K., Maki, T., Wood, M.L., and Monaco, A.P. 1995. Induction of tolerance to skin allografts by intrathymic injection of donor splenocytes. Transplantation. 60:1268-1273.

7. Goss, J.A., Nakafusa, Y., Yu, S., and Flye, M.W. 1993. Intrathymic injection of donor alloantigens induces specific tolerance to cardiac allografts. Transplantation. 56:166-173.

8. Ardavin, C. 1997. Thymic dendritic cells. Immunol. Today. 18:350-361.

9. de St. Groth, B.F. 1998. The evolution of self-tolerance: a new cell arises to meet the challenge of self-reactivity. Immunol. Today. 19:448-454.

10. Oluwole, S.F., et al. 1991. Migration patterns of dendritic cells in the rat: comparison of the effects of $\gamma$ and UV-B irradiation on the migration of dendritic cells and lymphocytes. Cell. Immunol. 133:390-407.

11. Kupiec-Weglinski, J.W., Austyn, J.M., and Morris, P.J. 1988. Migration patterns of dendritic cells in the mouse. J. Exp. Med. 167:632-645.

12. Lappin, M.B., et al. 1999. Analysis of mouse dendritic cell migration in vivo upon subcutaneous and intravenous injection. Immunology. 98:181-186.

13. Simoneau-Robin, I., Mousson, C., Zanetta, G., Racadot, E., and Rifle, G. 1998. In vitro study of alloreactivity and microchimerism after injection of dendritic cells and anti-CD4 monoclonal antibody in a combination of Lewis-Wistar Furth rats: preliminary data. Transplant. Proc. 30:2857-2858.

14. Delaney, J.R., Sykulev, Y., Eisen, H.N., and Tonegawa, S. 1998. Differences in the level of expression of class I major histocompatibility complex proteins on thymic epithelial and dendritic cells influence the decision of immature thymocytes between positive and negative selection. Proc. Natl. Acad. Sci. USA. 95:5235-5240.

15. Swiggard, W.J., Nonacs, R.M., Witmer-Pack, M.D., and Steinman, R.M. 1992. Enrichment of dendritic cells by plastic adherence and EA rosetting. In Current protocols in immunology. J.E. Coligan, A.M. Kruisbeek, D.H. 
Margulies, E.M. Shevach, and W. Strober, editors. John Wiley \& Sons Inc. New York, New York, USA. 3.7.1-3.7.11.

16. Hertz, M.I., Jessurun, J., King, M.B., Savik, S.K., and Murray, J.J. 1993. Reproduction of the obliterative bronchiolitis lesion after heterotopic transplantation of mouse airways. Am. J. Pathol. 142:1945-1951.

17. Neuringer, I.P., et al. 1998. Immune cells in a mouse model of obliterative bronchiolitis. Am. J. Respir. Cell Mol. Biol. 19:379-386.

18. Boehler, A., et al. 1997. Lymphocytic airway infiltration as a precursor to fibrous obliteration in a rat model of bronchiolitis obliterans. Transplantation. 64:311-317.

19. Rosenberg, A.S. 1992. Skin allograft rejection. In Current protocols in immunology. J.E. Coligan, A.M. Kruisbeek, D.H. Margulies, E.M. Shevach, and W. Strober, editors. John Wiley \& Sons Inc. New York, New York, USA. 4.4.1-4.4.12.

20. Dembic, Z., et al. 1985. Inbred and wild mice carry identical deletions in the E alpha MHC genes. EMBO J. 4:127-131

21. Duncan, S.R., et al. 1996. T cell receptor biases and clonal proliferations among lung transplant recipients with obliterative bronchiolitis. J. Clin. Invest. 97:2642-2650.

22. Suss, G., and Shortman, K. 1996. A subclass of dendritic cells kills CD4 T cells via Fas/Fas-ligand-induced apoptosis. J. Exp. Med. 183:1789-1796.

23. Kronin, V., et al. 1997. Are $\mathrm{CD}^{+}$dendritic cells (DC) veto cells? The role of CD8 on DC in DC development and in the regulation of CD4 and CD8 T cell responses. Int. Immunol. 9:1061-1064.

24. Pulendran, B., et al. 1999. Distinct dendritic cell subsets differentially regulate the class of immune response in vivo. Proc. Natl. Acad. Sci. USA 96:1036-1041

25. Goldrath, A.W., and Bevan, M.J. 1999. Low-affinity ligands for the TCR drive proliferation of mature CD8+ T cells in lymphopenic hosts. Immunity. 11:183-190

26. Krensky, A.M., Weiss, A., Davis, M.M., and Parham, P. 1990. T-lymphoycte-antigen interactions in transplant rejection. N. Engl. J. Med. 322:510-517.

27. Marrack, P., et al. 1993. The bacterial and mouse mammary tumor virus superantigens; two different families of proteins with the same functions. Immunol. Rev. 131:79-92.

28. Ardavin, C., et al. 1996. Expression and presentation of endogenous mouse mammary tumor virus superantigens by thymic and splenic dendritic cells and B cells. J. Immunol. 157:2789-2794

29. Moore, N.C., Anderson, G., McLoughlin, D.E., Owen, J.J., and Jenkinson, E.J. 1994. Differential expression of Mtv loci in MHC class II-positive thymic stromal cells. J. Immunol. 152:4826-4831.

30. Jarvis, C.D., Germain, R.N., Hager, G.L., Damshroder, M., and Mathis, L.A. 1994. Tissue-specific expression of messenger RNAs encoding endogenous viral superantigens. J. Immunol. 152:1032-1038.

31. Webb, S., Okamoto, A., Ron, Y., and Sprent, J. 1989. Restricted tissue distribution of Mls ${ }^{\mathrm{a}}$ determinants. J. Exp. Med. 169:1-12.

32. Rissoan, M.C., et al. 1999. Reciprocal control of T helper cell and dendritic cell differentiation. Science. 283:1183-1186.

33. Maldonado-Lopez, R., et al. 1999. CD $8 \alpha^{+}$and CD $8 \alpha^{-}$subclasses of dendritic cells direct the development of distinct T helper cells in vivo. J. Exp. Med. 189:587-592.

34. Stumbles, P.A., et al. 1998. Resting respiratory tract dendritic cells preferentially stimulate $\mathrm{T}$ helper cell type 2 (Th2) responses and require obligatory cytokine signals for induction of Th1 immunity. J. Exp. Med. 188:2019-2031.
35. Smith, A.L., and de St. Groth, B.F. 1999. Antigen-pulsed CD8 $\alpha^{+}$dendritic cells generate an immune response after subcutaneous injection without homing to the draining lymph node. J. Exp. Med. 189:593-598.

36. Grabbe, S., Kampgen, E., and Schuler, G. 2000. Dendritic cells: multi-lineal and multi-functional. Immunol. Today. 21:431-433.

37. Traver, D., et al. 2000. Development of CD $8 \alpha$-positive dendritic cells from a common myeloid progenitor. Science. 290:2152-2154.

38. Khoury, S.J., et al. 1995. Mechanisms of acquired thymic tolerance in experimental autoimmune encephalomyelitis: thymic dendriticenriched cells induce specific peripheral $\mathrm{T}$ cell unresponsiveness in vivo. J. Exp. Med. 182:357-366.

39. Matzinger, P., and Guerder, S. 1989. Does T-cell tolerance require a dedicated antigen-presenting cell? Science. 338:74-76.

40. Swat, W., Ignatowicz, L., von Boehmer, H., and Kisielow, P. 1991. Clonal deletion of immature CD4+CD8+ thymocytes in suspension culture by extrathymic antigen-presenting cells. Nature. 351:150-153.

41. Peugh, W.N., Austyn, J.M., Carter, N.P., Wood, K.J., and Morris, P.J. 1987. Inability of dendritic cells to prevent blood transfusion effect in a mouse cardiac allograft model. Transplantation. 44:706-711.

42. Larsen, C.P., Barker, H., Morris, P.J., and Sustyn, J.M. 1990. Failure of mature dendritic cells of the host to migrate from the blood into cardiac or skin allografts. Transplantation. 50:294-301.

43. Huang, A.Y.C., et al. 1994. The role of bone marrow-derived cells in presenting class I-restricted tumor antigens. Science. 264:961-965.

44. Chen, W., Sayegh, M.H., and Khoury, S.J. 1998. Mechanisms of acquired thymic tolerance in vivo: intrathymic injection of antigen induces apoptosis of thymocytes and peripheral $\mathrm{T}$ cell anergy. J. Immunol. 160:1504-1508.

45. Kisielow, P., Bluthmann, H., Staerz, U.D., Steinmetz, M., and von Boehmer, H. 1988. Tolerance in T-cell-receptor transgenic mice involves deletion of nonmature CD4+CD8+ thymocytes. Nature. 333:742-746.

46. Bouneaud, D., Kourilsky, P., and Bousso, P. 2000. Impact of negative selection on the $T$ cell repertoire reactive to a self-peptide: a large fraction of T cell clones escapes clonal deletion. Immunity. 13:829-840.

47. Oehen, S., Feng, L., Xia, Y., Surh, C.D., and Hedrick, S.M. 1996. Antigen compartmentation and T helper cell tolerance induction. J. Exp. Med. 183:2617-2626.

48. Qin, S., et al. 1993. "Infectious" transplantation tolerance. Science. 259:974-977.

49. Gonzalez-Quintial, R., and Theofilopoloulos, A.N. 1992. V $\beta$ repertoires in aging mice. J. Immunol. 149:230-236.

50. Gorski, J., Yassai, M., Keever, C., and Flomberg, N. 1995. Analysis of reconstituting $\mathrm{T}$ cell receptor repertoires in bone marrow transplant recipients. Arch. Immunol. Ther. Exp. 43:93-97.

51. Sykes, M. 1996. Immunobiology of transplantation. FASEB J. 10:721-730.

52. Saunders, D., et al. 1996. Dendritic cell development in culture from thymic precursor cells in the absence of granulocyte/macrophage colony-stimulation factor. J. Exp. Med. 184:2185-2196.

53. Ohnishi, K., Daigo, M., and Tokunaga, T. 1995. SV40-adenovirus immortalized cell lines derived from mouse lymphoid dendritic cell preparations. Immunol. Cell Biol. 73:205-211.

54. Bjorck, P., Banchereau, J., and Flores-Romo, L. 1997. CD40 ligation counteracts Fas-induced apoptosis of human dendritic cells. Int Immunol. 9:365-372. 